\title{
Automated discrete electron tomography - Towards routine high-fidelity reconstruction of nanomaterials
}

\author{
Xiaodong Zhuge $^{\mathrm{a}, *}$, Hiroshi Jinnai ${ }^{\mathrm{b}}$, Rafal E. Dunin-Borkowski ${ }^{\mathrm{c}}$, Vadim Migunov ${ }^{\mathrm{c}}$, Sara Bals ${ }^{\mathrm{d}}$, \\ Pegie $\mathrm{Cool}^{\mathrm{e}}$, Anton-Jan Bons ${ }^{\mathrm{f}}$, Kees Joost Batenburg ${ }^{\mathrm{a}}$ \\ a Computational Imaging, Centrum Wiskunde \& Informatica, Science park 123, 1098XG Amsterdam, Netherlands \\ b Institute of Multidisciplinary Research for Advanced Materials, Tohoku University, Katahira 2-1-1, Aoba-ku, Sendai 980-8577, Japan \\ ${ }^{\mathrm{c}}$ Ernst Ruska-Centre for Microscopy and Spectroscopy with Electrons and Peter Grünberg Institute, Forschungszentrum Jülich, D-52425 Jülich, Germany \\ d EMAT, University of Antwerp, Groenenborgerlaan 171, B-2020 Antwerp, Belgium \\ e Laboratory of Adsorption and Catalysis, Department of Chemistry, University of Antwerp, Universiteitsplein 1, 2610 Wilrijk, Belgium \\ ${ }^{\mathrm{f}}$ European Technology Center, ExxonMobil Chemical Europe Inc., Hermeslaan 2, B-1831 Machelen, Belgium
}

\section{A R T I C L E I N F O}

\section{Keywords:}

Discrete Tomography

Electron Tomography

Missing-Wedge Artifacts

Transmission Electron Microscopy

\begin{abstract}
A B S T R A C T
Electron tomography is an essential imaging technique for the investigation of morphology and 3D structure of nanomaterials. This method, however, suffers from well-known missing wedge artifacts due to a restricted tilt range, which limits the objectiveness, repeatability and efficiency of quantitative structural analysis. Discrete tomography represents one of the promising reconstruction techniques for materials science, potentially capable of delivering higher fidelity reconstructions by exploiting the prior knowledge of the limited number of material compositions in a specimen. However, the application of discrete tomography to practical datasets remains a difficult task due to the underlying challenging mathematical problem. In practice, it is often hard to obtain consistent reconstructions from experimental datasets. In addition, numerous parameters need to be tuned manually, which can lead to bias and non-repeatability. In this paper, we present the application of a new iterative reconstruction technique, named TVR-DART, for discrete electron tomography. The technique is capable of consistently delivering reconstructions with significantly reduced missing wedge artifacts for a variety of challenging data and imaging conditions, and can automatically estimate its key parameters. We describe the principles of the technique and apply it to datasets from three different types of samples acquired under diverse imaging modes. By further reducing the available tilt range and number of projections, we show that the proposed technique can still produce consistent reconstructions with minimized missing wedge artifacts. This new development promises to provide the electron microscopy community with an easy-to-use and robust tool for high-fidelity $3 \mathrm{D}$ characterization of nanomaterials.
\end{abstract}

\section{Introduction}

Increasing interest in the modeling and development of advanced nanomaterials has fueled the demand for optimized imaging methods capable of accurate characterization of such systems. Electron tomography (ET) is an important and powerful technique for the investigation of the three-dimensional structures of nanomaterials [1,2], and has been widely applied to materials that include polymeric structures $[3,4]$, inorganic materials [5,6] and organic materials [7].

Despite recent advances in instrumentation and automated image acquisition, ET is fundamentally limited by the accuracy of the reconstructions it can produce, largely due to the well-coined 'missing wedge' problem [8]. For most types of samples, the maximum tilt range, over which $2 \mathrm{D}$ projection images are acquired, is restricted to approximately $\pm 70^{\circ}$ (and sometimes less in practice) due to an increase in the effective thickness of a thin section at high tilt angles, shadowing by the sample grid and sample holder, and limited space between the polepieces of the objective lens in the transmission electron microscope (TEM). This limited tilt range results in an unavoidable missing gap of information in the Fourier representation of the reconstruction, which can lead to false vertical elongation of structures and to the disappearance of horizontal features. As a result, segmentation of the reconstructions needs to be performed manually to reduce the influence of artifacts, which is not only time-consuming but also affects the objectiveness and repeatability of quantitative structural analysis.

Significant efforts have been made by the electron microscopy community to address the missing wedge problem. Dual-axis ET is an

\footnotetext{
* Corresponding author.
} 
alternative acquisition strategy, which involves the acquisition of additional projection images about a second tilt axis that is perpendicular to the original one $[9,10]$. Although this approach can partially reduce artifacts by decreasing the lost information to a 'missing pyramid', the doubled electron dose and difficulty in acquiring as well as aligning two tilt series add many complications. Needle-shaped specimens that have been prepared using focused ion beam (FIB) milling together with the use of a dedicated tomography holder, enable the acquisition of projection images over the complete tilt range of $180^{\circ}$, thereby eliminating missing wedge artifacts [11-13]. However, not all samples can be prepared and imaged using this method and the diameter of the specimen that one can image is also limited.

The development of advanced reconstruction algorithms is another important direction for alleviating the missing wedge problem. Weighted backprojection (WBP) [14] and iterative reconstruction algorithms, such as the simultaneous iterative reconstruction technique (SIRT) [15], are now widely used but do not offer solutions to the missing wedge problem. Furthermore, their performance degrades significantly when the number of projection images is limited. Compressive sensing ET (CS-ET) represents a type of algorithm that has undergone intensive research in recent years $[16,17]$. According to CS theory, if an image is sparse in a certain domain then it can be recovered accurately from a small number of measurements with high probability when the measurements satisfy certain randomization properties [18]. In several cases, it has been demonstrated that artifacts due to limited sampling in conventional reconstructions could be reduced by selecting proper sparsifying transforms [17]. Recent studies have shown that the combination of CS-ET with the use of a needleshaped specimen can provide high-fidelity reconstructions from fewer projections [12]. Total Variation minimization (TVmin) can be seen as a special case of CS-ET when the boundary representing the interface between different compositions is sparse [19,20].

Discrete tomography (DT) makes use of a type of prior knowledge, where the sample is known to consist of a limited number of materials [21]. Historically, DT was first formulated for the reconstruction of nanocrystals at atomic resolution in samples that contain only a few types of atoms that also lie on regular lattices [22]. In more generalized cases, where individual atoms cannot be resolved, DT assumes that the reconstruction contains a few different intensities, which correspond to a limited number of materials, with no assumption about the lattice structure. By exploiting this prior knowledge, DT has the potential to provide a significant reduction in missing wedge artifact, as well as in the number of projection images required and exposure of the specimen.

The Discrete Algebraic Reconstruction Technique (DART), which is one of the practical algorithms for DT [23], has demonstrated its ability to improve the reconstruction quality in several practical cases [2428]. However, the application of DART to practical tilt series remains a difficult and often time-consuming task. Several key issues must be addressed if it is to reach a general level of applicability to nanomaterials. First, DART requires very strict conditions on the discreteness of the reconstruction, which are not fulfilled in all tomographic acquisition modes in the TEM. In fact, all previous results have been demonstrated using HAADF-STEM mode, where the projection requirement for tomography is better fulfilled. Other imaging modes, such as BF-TEM, have their own advantages in applications for the study of beam sensitive materials such as polymers or meso-porous structures. Second, just as for many other DT algorithms, DART is sensitive to noise in the projection data and can produce unstable artifacts such as rough edges and salt-and-pepper noise in the resulting reconstruction. Last but not least, one must specify numerous parameters for DART, including the discrete grey levels that correspond to each composition, in order to achieve reasonable results. This requires a high level of algorithm-specific expertise from the user and may involve trial-and-error, which limits the wide adoption of the technique.
In this paper, we present a recently proposed technique, named TVR-DART [29], which addresses all of the above-mentioned problems by incorporating a flexible and relaxed discrete model, while exploiting two types of prior information simultaneously in terms of limited material composition and sparsity of boundaries inspired by compressive sensing. An improved 3D version of the technique is introduced in this paper, which allows us to expand the application of DT to different types of materials for various TEM acquisition modes, thereby improving the fidelity of the reconstruction and its automation.

The key concepts and formulations of the proposed technique are described in Section 2. In Section 3, we present the application of TVRDART to three different experimental electron tomography datasets that were obtained using diverse imaging modes and acquisition conditions. By using a needle-shaped specimen, we demonstrate the extended application of DT to BF-TEM images, and compare the TVRDART reconstruction under a $\pm 60^{\circ}$ tilt range with the one obtained from a complete dataset $\left( \pm 90^{\circ}\right)$. The second dataset of an inorganic nanotube was recorded on a direct electron detector and has a high level of noise due to the extremely short exposure time (the complete tilt series recorded in under $3.5 \mathrm{~s}$ ). We use this dataset to show that rough edges, which appear in the reconstructions obtained using the original DART algorithm as a result of high noise level, are now effectively suppressed, allowing accurate reconstruction despite the use of an extremely limited tilt range of $\pm 50^{\circ}$. The last dataset is a tilt series of an anatase nanosheet specimen recorded using HAADFSTEM. We demonstrate with this data that challenging horizontal structures, which usually disappear due to missing wedge artifacts, can be recovered using the proposed technique. Section 4 discusses the results and concludes the paper.

\section{Methods}

\subsection{Algorithm}

For a limited angular range and large tilt increments, the mathematical problem of tomographic reconstruction is highly underdetermined based on only the acquired data. This situation results in the need to fully utilize any prior knowledge we have about the underlying specimen. On the assumption that the total number of different material compositions within the sample is known a priori, the proposed TVR-DART technique exploits explicitly two types of priors: sparsity of image grey levels (each corresponding to a distinct material composition) and sparsity of boundaries between different compositions. The first type of sparsity promotes a solution that has a smaller amount of different grey levels, while the second type helps to minimize the amount of noise within the reconstruction. The two types of priors are incorporated in different mathematical formats and can be solved within the following optimization framework:

$$
\hat{\boldsymbol{x}}=\underset{\boldsymbol{x}, \quad \bar{R}}{\arg \min }\left\{\|\boldsymbol{W S}(\boldsymbol{x}, \bar{R})-\boldsymbol{p}\|_{2}^{2}+\lambda\|\nabla[S(\boldsymbol{x}, \bar{R})]\|_{1}\right\}
$$

where $\boldsymbol{x}$ is a voxel representation of the specimen, $\boldsymbol{p}$ is the acquired tilt series, $\boldsymbol{W}$ is the projection matrix that maps the reconstruction to the measured projection data, $S(\boldsymbol{x}, \bar{R})$ represents a Soft Segmentation Function (SSF) that smoothly steers the intensities of the reconstruction towards a set of discrete grey levels $\bar{R}=\left\{\rho_{0}, \rho_{1}, \ldots, \rho_{\mathrm{G}}\right\}$ with $\mathrm{G}$ being the total number of different material compositions besides vacuum, and $\nabla$ represents the discrete gradient operator. The first $\ell^{2}$-norm term in Eq. (1) ensures a match between the reconstruction after segmentation and the projection data while the second $\ell^{1}$-norm promotes sparsity in boundaries between the segmented regions of different materials. The parameter $\lambda$ is the weight for controlling the trade-off between the two terms. Inclusion of the SSF in the objective function applies a soft push to the pixel values that encourages (but not strictly enforces) a discrete solution. As a result of this flexibility, TVR-DART exhibits extra tolerance towards miss-matches in the projection data, 


\section{Bilevel Optimization}

\section{Outer layer - reconstruction:}

$\widehat{\boldsymbol{x}}^{t+1}=\underset{\boldsymbol{x}}{\arg \min }\left\{\left\|\boldsymbol{W S}\left(\boldsymbol{x}, \bar{R}^{t+1}\right)-\boldsymbol{p}\right\|_{2}^{2}+\lambda\left\|\nabla\left[S\left(\boldsymbol{x}, \bar{R}^{t+1}\right)\right]\right\|_{1}\right\}$

Inner layer - grey level estimation:

$\bar{R}^{t+1}=\underset{\bar{R}}{\arg \min }\left\{\left\|\boldsymbol{W} S\left(\widehat{\boldsymbol{x}}^{t}, \bar{R}\right)-\boldsymbol{p}\right\|_{2}^{2}+\lambda\left\|\nabla\left[S\left(\widehat{\boldsymbol{x}}^{t}, \bar{R}\right)\right]\right\|_{1}\right\}$

Subject to $\mathrm{G}$ total number of discrete grey levels

Fig. 1. Illustration of the bilevel optimization framework of TVR-DART for joint estimation of reconstruction and grey levels subject to a specified number of different material compositions.

e.g., noise and less-than-ideal imaging conditions. A dedicated algorithm that forms auxiliary functions above the second order approximation of the objective function was formulated to efficiently solve the underlying non-convex optimization problem. Detailed formulations of the algorithm can be found in Ref. [29].

With the objective function formulated in Eq. (1), both the reconstruction $\hat{\boldsymbol{x}}$ and the set of discrete grey levels $\bar{R}$ corresponding to each of the compositions must be optimized or determined. Due to the different nature of the two types of unknowns, the problem is solved in a manner of bilevel optimization: the optimization on the grey levels $\bar{R}$ is treated as an inner layer problem, which nests within an outer layer optimization on the reconstruction $\hat{\boldsymbol{x}}$. This approach is illustrated in Fig. 1. Within every iteration of the algorithm, a converged solution for the grey levels $\bar{R}^{t+1}$ is first found using the current estimate $\hat{\boldsymbol{x}}^{t}$, before making a step of the outer layer problem to find the next estimate of the reconstruction $\hat{\boldsymbol{x}}^{t+1}$. Efficient second-order algorithms can be used to solve the inner optimization of grey levels [29]. The whole algorithm alternates between the inner and outer optimization tasks until the convergence is reached.

We further propose a modified formulation for the SSF function of TVR-DART as a sum of generalized logistic functions (from previous logistic function):

$S(\hat{\boldsymbol{x}}, \bar{R})=\rho_{0}+\sum_{g=1}^{\mathrm{G}} \frac{\left(\rho_{g}-\rho_{g-1}\right)}{\left[\mathbf{1}+\boldsymbol{e}^{-2 k_{g}\left(\hat{\boldsymbol{x}}-\tau_{g}\right)}\right]^{v_{g}}}$

where $\rho_{g}$ are the discrete grey levels with $\rho_{0}=0$ (assuming the background is always vacuum, corresponding to zero intensity in the reconstruction), $\tau_{g}$ represent the corresponding thresholds, and $k_{g}$ controls the sharpness of the SSF. The variable $v_{g}$ in Eq. (2) is introduced to help ensure that pixels with intensities below $\tau_{g}$ are pushed downwards, while those above $\tau_{g}$ are pushed upwards.

The TVR-DART algorithm starts with an initial solution for the reconstruction using either SIRT or Total Variation minimization. For all of the results presented in this paper, an initial SIRT solution with 200 iterations is used. We further normalize the projection data with the maximum value of the initial SIRT reconstruction, so that the value range of the reconstruction lies approximately between 0 and 1 for all of the datasets. We also initialize the values of the set of grey levels $\bar{R}^{0}$ using the average pixel values within identified regions of different materials from the normalized initial reconstruction. A sample script demonstrating the workflow of applying TVR-DART on experimental datasets can be found in the appendix.

\subsection{Simulation}

In order to gain further insights into the proposed TVR-DART technique, a numerical tomography simulation was carried out using a simple linear projection model. Fig. 2 shows the reconstructions of a

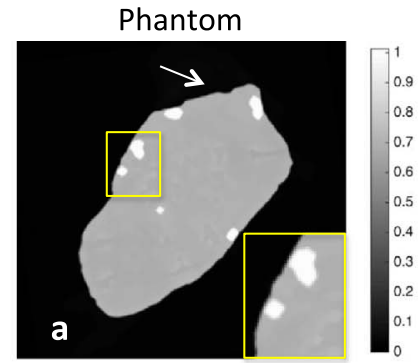

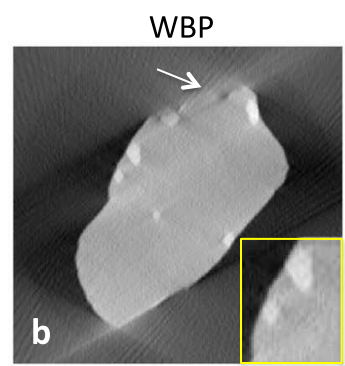

DART

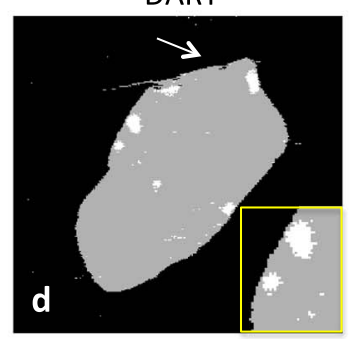

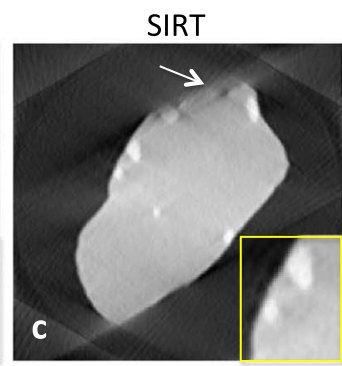

TVR-DART

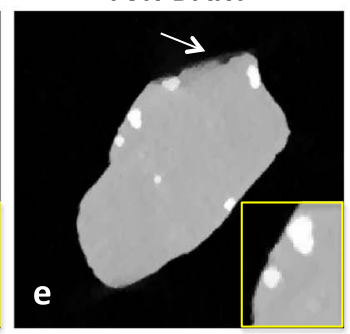

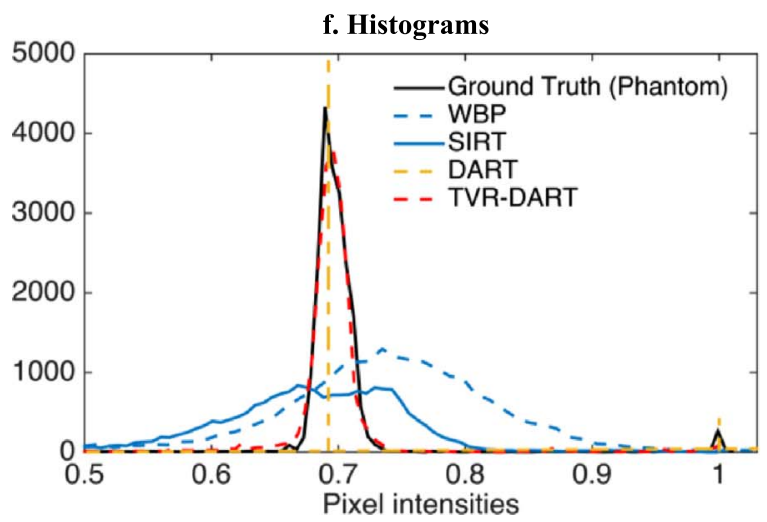

Fig. 2. Numerical tomography simulations of (a) a phantom with intensity gradients within two of its compositions and its reconstructions performed for a $\pm 60^{\circ}$ tilt range with $2^{\circ}$ increments obtained using (b) WBP, (c) SIRT, (d) DART and (e) TVR-DART. Comparison of histograms (f) of the original phantom and reconstructions obtained using different algorithms, showing the closest match between the TVR-DART reconstruction and the ground truth.

phantom, which resembles a specimen in which nanoparticles are embedded in a base matrix. The chosen phantom is not completely discrete, but contains intensity gradients within its two compositions. This mimics practical data within which non-ideal imaging conditions, such as non-monotonicity of the recorded contrast, are present. The simulated tilt range is $\pm 60^{\circ}$ with a $2^{\circ}$ tilt increment to represent a missing wedge condition. To make the simulation more realistic, Gaussian noise was also added to the projection data. Visual comparison of the reconstructions obtained from the different techniques shows that TVR-DART and DART provide more accurate reconstructions with reduced missing wedge artifacts, while DART further results in rough edges and a small amount of artifacts within the object. Fig. 2(f) further compares the histograms of different reconstructions 
Table 1

Parameter settings for the 3 experimental datasets.

\begin{tabular}{llll}
\hline & $\begin{array}{l}\text { Needle-shaped } \\
\text { polymer (BF-TEM) }\end{array}$ & $\begin{array}{l}\text { Inorganic nanotube } \\
\text { (BF-TEM) }\end{array}$ & $\begin{array}{l}\text { Anatase nanosheets } \\
\text { (HAADF-STEM) }\end{array}$ \\
\hline$K$ & 4 & 4 & 4 \\
$r$ & 0.5 & 0.5 & 0.5 \\
$\lambda$ & 150 & 10 & 40 \\
\hline with $k_{g}=\frac{K}{\rho_{g}-\rho_{g-1}}$, and $v_{g}=-\log _{2} r$ &
\end{tabular}

with the ground truth. Conventional techniques, such as WBP and SIRT, produce blurred edges under missing wedge conditions, which are represented as spread intensities and a widened histogram distribution. In contrast, DART enforces a completely discrete solution which forces the reconstruction to contain only 2 grey levels besides vacuum. Under less ideal imaging conditions, morphological artifacts around the edge and inner regions of the object are introduced in order to provide a match with the projection data, while following this strict discrete assumption. The histogram of TVR-DART reconstruction matches with the ground truth well, as it concentrates the grey levels towards a limited number of grey values but does not strictly enforce this criterion. It thereby allows an additional degree of tolerance towards small mismatches in the acquired data, while still being able to take advantage of the strong discrete assumption.

\subsection{Parameters}

For the three experimental datasets described in the next section, we specify the parameter settings in the algorithm that are listed in Table 1. Parameter $K$ is a transition constant for controlling the sharpness of transitions in the SSF curve, while $r=\left(\tau_{g}-\rho_{g-1}\right) /\left(\rho_{g}-\rho_{g-1}\right)$ determines the relative position of the thresholds between adjacent grey levels.

For all three datasets, only $\lambda$ is set differently, depending on the noise level and the imaging mode of the experiment. The fact that the same settings can be applied to different datasets and imaging conditions, with the exception of a single parameter, demonstrates the automation of the proposed technique.

Three algorithms are further used for comparison in this study: WBP, SIRT, and DART. For (additive) SIRT, 200 iterations are used to ensure convergence. For the DART implementation, SIRT is utilized as the intermediate algebraic reconstruction step. The algorithm iterates for 100 iterations to ensure convergence. Within each main iteration, 20 sub-iterations of SIRT are performed on the free pixels (pixels that are allowed to change). In order to maximize its ability to cope with noise in the projection data, the fix probability (the percentage of pixels that are fixed during update) is specified as $p=0.5$ as described in Ref. [23]. A Gaussian filter of a size $3 \times 3$ with $\sigma=0.6$ is used to smooth the boundary pixels after each iteration. Because DART requires further input on the grey levels of the compositions, we use the values estimated by TVR-DART to assist the DART reconstructions.

\section{Experimental results from three case studies}

In this section, we report the electron tomography experiments carried out for three different types of specimens in materials science imaged using different TEM acquisition modes. For every sample, descriptions of the composition, preparation and acquisition conditions are given, followed by an analysis of results from different reconstruction techniques.

A Structural Similarity Index (SSIM) [30] is used to evaluate the quality of the reconstructions. This is a quantitative measure of the similarity between the reconstruction obtained from a limited (reduced) dataset and one computed from the full available tilt series. A perfect reconstruction should produce an SSIM that is close to 1 (maximum). We also compute the projection distance measure using the $\ell^{2}$-norm $\left(\|\boldsymbol{W} \boldsymbol{x}-\boldsymbol{p}\|_{2}\right)$ as an estimate of the match between the reconstructions and the projection data. The lower the projection distance measure, the better the consistency of the reconstruction with the acquired tilt series.

\subsection{Needle-shaped nanocomposite acquired with BF-TEM}

A polymer nanocomposite was prepared by a direct dispersion method from ultrafine zirconium dioxide (zirconia) particles and a thermally stable polymer. A FIB system (FB2100, Hitachi, Ltd., Japan) was used to make a needle-shaped TEM specimen from the nanocomposite. The diameter of the resulting needle was approximately $150 \mathrm{~nm}$ at the tip and $300 \mathrm{~nm}$ at the base. The grid with the rod-shaped specimen at the top was mounted on a dedicated specimen holder to achieve a $\pm 90^{\circ}$ tilt range. Electron tomography experiment was carried out using a JEM2200FS TEM (JEOL Ltd., Japan) equipped with a eucentric specimen stage. The accelerating voltage was $200 \mathrm{kV}$. In total, 180 bright-field projection images from $-90^{\circ}$ to $+90^{\circ}$ with $1^{\circ}$ increments were acquired using a slow-scan charge-coupled device (CCD) camera (Gatan USC1000). The pixel size of the acquired images was $0.45 \mathrm{~nm}$. The tilt series of the electron micrographs was aligned by using the zirconia grains as fiducial markers. Full details of the experiment can be found in Ref. [11]. Fig. 3 shows a projection image of the needle-shaped specimen. The $5-20 \mathrm{~nm}$ zirconia grains are visible as dark domains in the polymer matrix in the enlarged region. Before reconstruction, a pixel-wise logarithm, followed by a negation, were applied to the data, based on the assumption of image formation according to the Beer-Lambert law. This is a necessary step before reconstruction in making the projection image intensity linearly proportional to the material thickness. Finally, an intensity-offset correction was applied so that the average value of the visible background was zero (corresponding to vacuum).

The challenge in applying discrete tomography to a BF-TEM tilt series results from its complex contrast mechanism where image intensities are affected by both amplitude and phase contrast. This means that the projection requirement, by which image intensities must vary monotonically with material thickness, is hard to guarantee in BF-TEM mode. A single composition in the sample then does not produce a single grey level in the reconstruction. Instead, pixel intensities tend to spread around certain grey levels, which violates the strict assumption of a conventional discrete tomography algorithm.

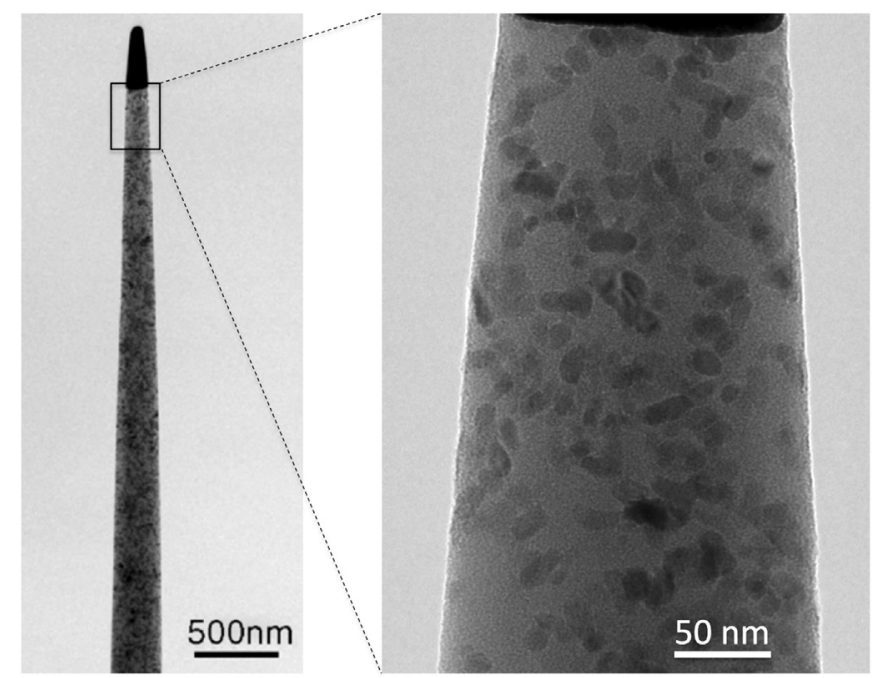

Fig. 3. BF-TEM image of the needle-shaped polymer nanocomposite and an enlarged region of the thinnest part of the specimen showing dark regions corresponding to 5$20 \mathrm{~nm}$ zirconia grains. 

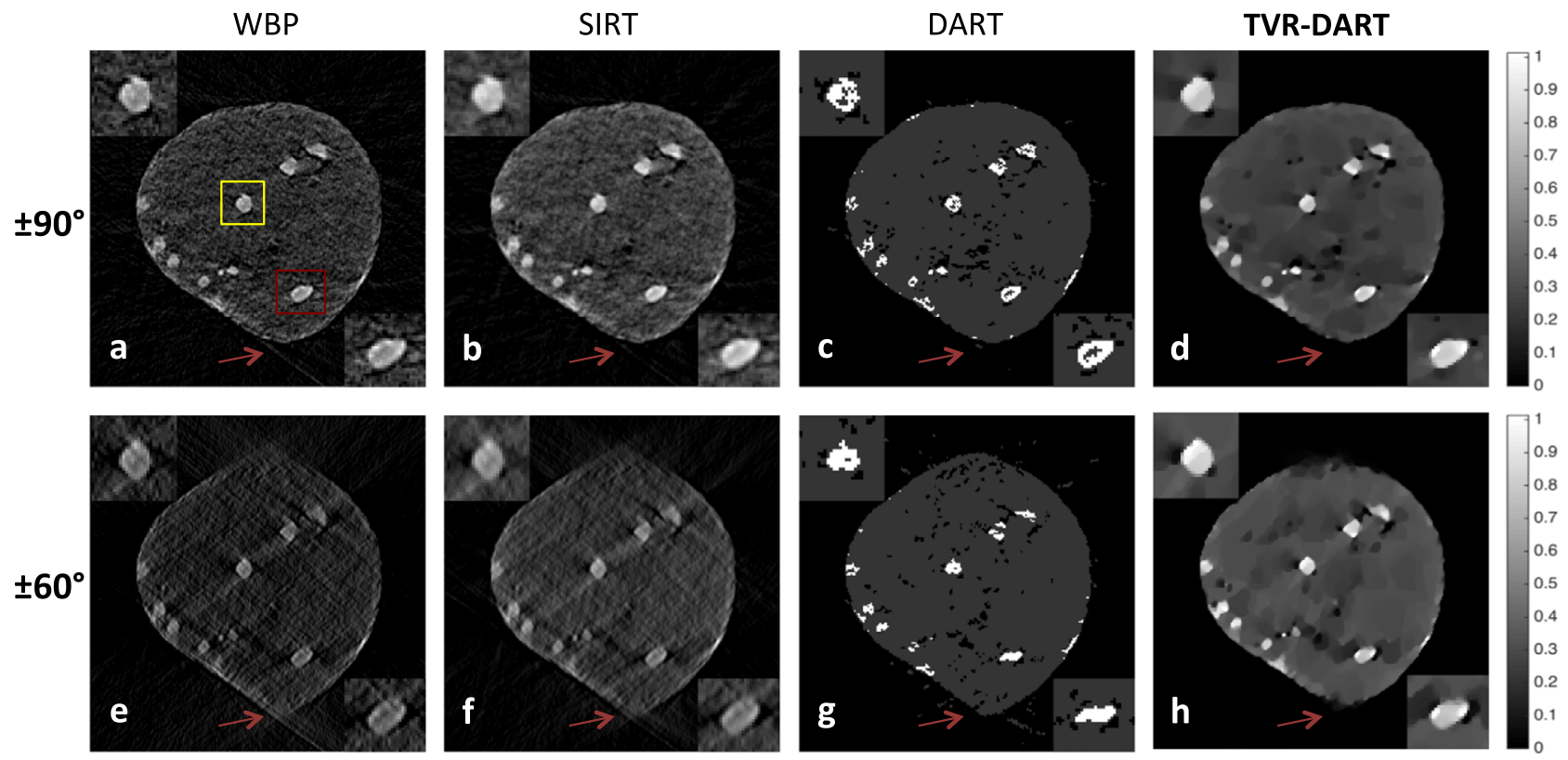

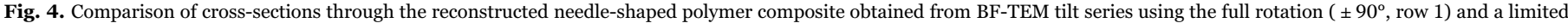

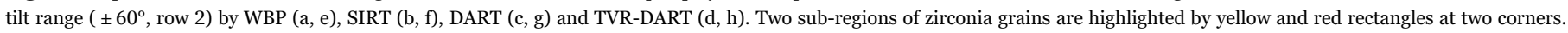

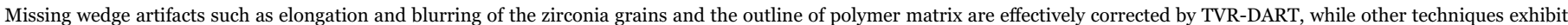
different types of artifacts.

The proposed TVR-DART technique can then succeed due to its flexible prior model. Fig. 4 shows cross-sections through the reconstructed needle-shaped polymer composite obtained using WBP, SIRT, DART and TVR-DART. The availability of full rotation of the needle-shaped specimen provides the opportunity to further verify the fidelity of reconstructions obtained using a limited tilt range. We computed reconstructions from a reduced tilt series with a $\pm 60^{\circ}$ tilt range (120 projections) and compared them with reconstructions computed from the full dataset. Two sub-regions of zirconia grains are highlighted by yellow and red boxes at two corners. Both WBP and SIRT result in typical missing wedge artifacts. DART tries to force the reconstruction to be completely discrete (with only two grey levels besides zero), causing unstable features to appear within the polymer matrix and zirconia grains. Artifacts such as vertical elongation and blurring of the zirconia grains and polymer matrix are effectively reduced by using TVR-DART.

By tolerating a small amount of intensity gradient to exist within each of the two compositions, the reconstruction becomes more consistent, despite the fact that the discrete assumption is not completely fulfilled. It is worth noting that the grey levels were

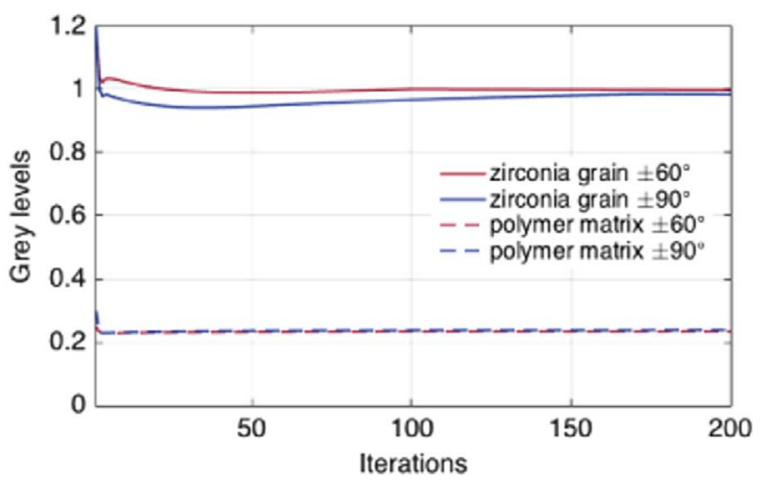

Fig. 5. Automated grey level estimation for both the zirconia grains and the polymer matrix within the polymer nanocomposite by TVR-DART using full rotation $\left( \pm 90^{\circ}\right)$ and a limited tilt range $\left( \pm 60^{\circ}\right)$. For both material compositions, the estimated grey levels under a reduced angular range are similar to those estimated from the full tilt series. estimated independently for both the full and reduced tilt ranges (Fig. 5), yet they still converged to similar values for the two compositions in the two separate reconstructions. Both the SSIM and the projection distance of the reconstructions are shown in Fig. 6. The TVR-DART reconstruction for the $\pm 60^{\circ}$ tilt range results in a SSIM of 0.953, which is the most consistent of the values obtained using different techniques with the reconstruction obtained using full rotation. Its projection distance is also the lowest (by an order of magnitude), indicating that it provides the most compliant reconstruction with the original projection data.

\subsection{Lanthanide-based inorganic nanotube recorded using a direct electron detector}

Inorganic lanthanide nanotubes were prepared from misfit-layered compounds in the forms of alternating crystallographic layers with different periodicities [31]. Specifically, LaS layers that have a rock-salt structure were alloyed with Ce alternate with layers of hexagonal $\mathrm{CrS}_{2}$. For the tomography experiment, nanotubes were dispersed onto lacey C grids. Tomographic tilt series were acquired using a FEI Titan $60-$

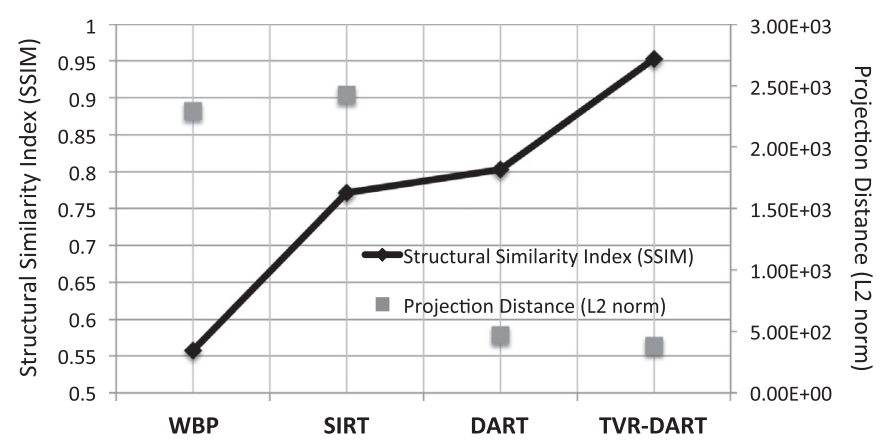

Fig. 6. Structural Similarity Index (SSIM) and Projection Distance $\left(\ell^{2}\right.$-norm) for reconstructions of the polymer nanocomposite from WBP, SIRT, DART and TVRDART under a $\pm 60^{\circ}$ tilt range. Corresponding reconstructions using the full tilt range ( $\pm 90^{\circ}$ ) are used as reference images for the calculation of the SSIM. TVR-DART shows the highest structural similarity (0.953), while exhibiting the best match with the projection data (lowest $\ell^{2}$-norm). 


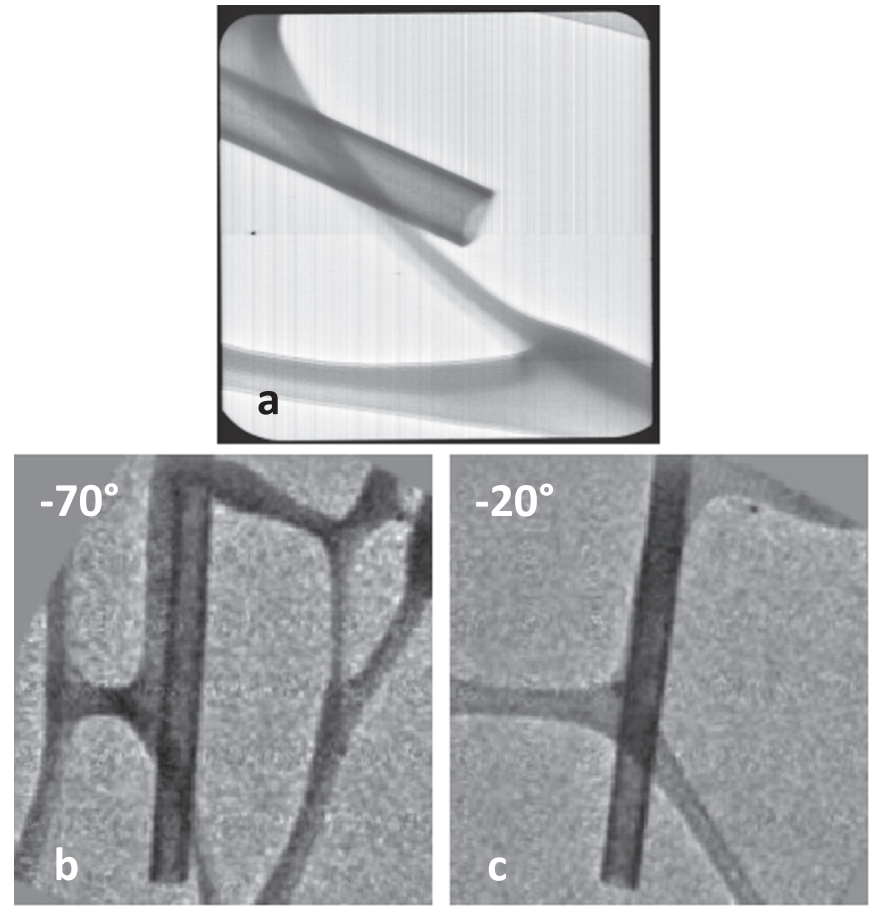

Fig. 7. BF-TEM images of an individual inorganic lanthanide nanotube on a lacey $\mathrm{C}$ grid. (a) High magnification electron micrograph of the apex of the nanotube showing regular tube structure, and examples of projection images for tilt angles of (b) $-70^{\circ}$ and (c) $-20^{\circ}$, taken from the complete $100^{\circ}$ tilt range, are shown.
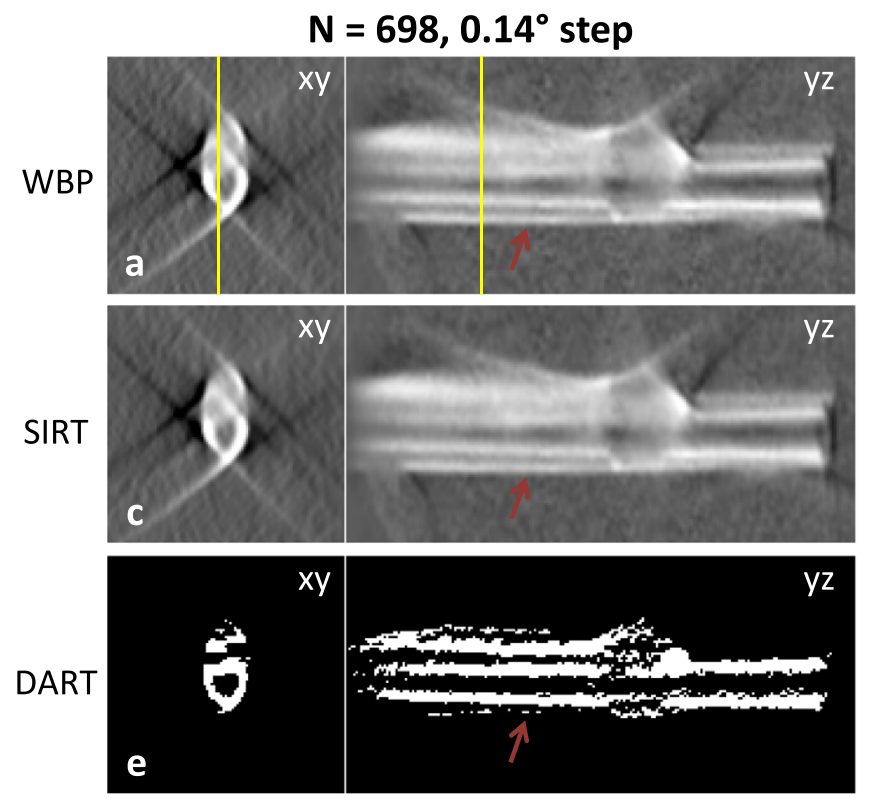

yz

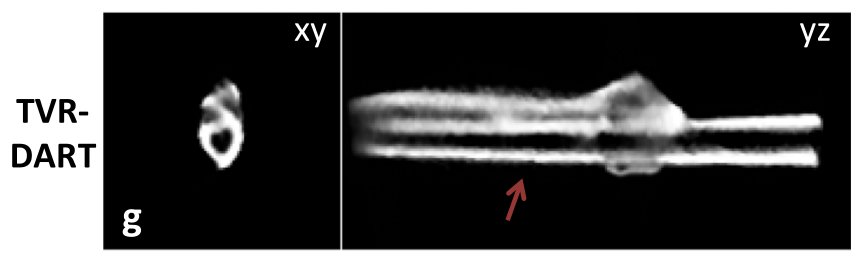

300 TEM operated at $60 \mathrm{kV}$ using a Fischione Model 2010 single-tilt tomography holder. A direct electron detection camera with a pnCCD sensor (PNDetector $\mathrm{GmbH}$ ) was mounted below the projection chamber and used to record BF-TEM images. A series of 3487 images with a pixel size of $5.32 \mathrm{~nm}$ was acquired during rotation of the specimen over a tilt range of $-70^{\circ}$ to $+30^{\circ}$ under continuous electron beam illumination, while keeping the majority parts of an individual nanotube in focus and within the field-of-view [32]. The entire tilt series was acquired with $1.34 \times 10^{5}$ electrons per frame, corresponding to approximately 2 electrons per pixel per frame. A reduced dataset consisting of an average of five neighboring projections (698 images) was used for reconstruction. The projection images were aligned with sub-pixel precision using an iterative feedback algorithm that optimizes the contrast and resolution of the tomogram. A high magnification image (2.63 $\mathrm{nm}$ pixel size) of the apex of the nanotube and two of the acquired projection images recorded at $-70^{\circ}$ and $-20^{\circ}$ (middle) are shown in Fig. 7. As in the previous polymer study, the negative logarithm of each image in the BF-TEM tilt series was computed, followed by intensity offset-correction, before performing tomographic reconstructions.

For this experimental dataset, the challenges relate mainly to the high noise level in the tilt series and the extremely limited tilt range. Fig. 8 shows cross-sections (xy- and yz-planes) through the center of the reconstructed nanotube obtained using different reconstruction techniques. Traditional algorithms such as WBP and SIRT require a large number of projections and suffer from missing wedge artifacts for such a limited tilt range. Their reconstructions in the yz-plane contain extra double layer structures outside the wall of the nanotube (indicated by the red arrow). Because this particular type of nanotube commonly exhibit regular wall wrapping [31], it is obvious that such double-wall structures are the result of missing wedge artifacts. In
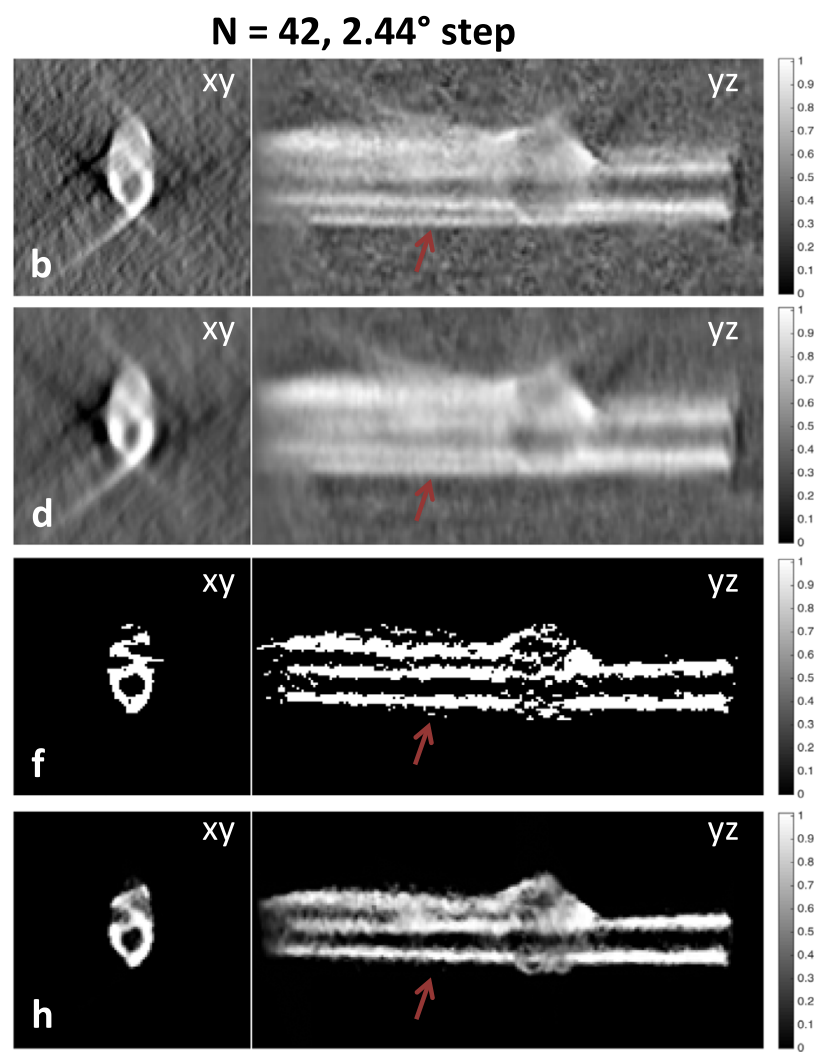

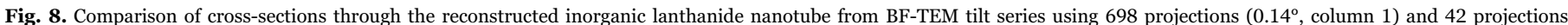

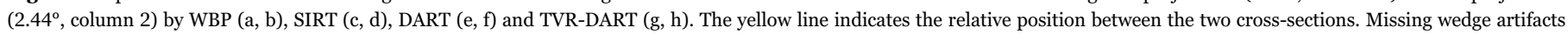

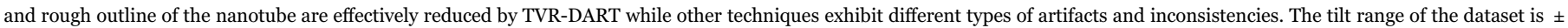
$50^{\circ}$. 


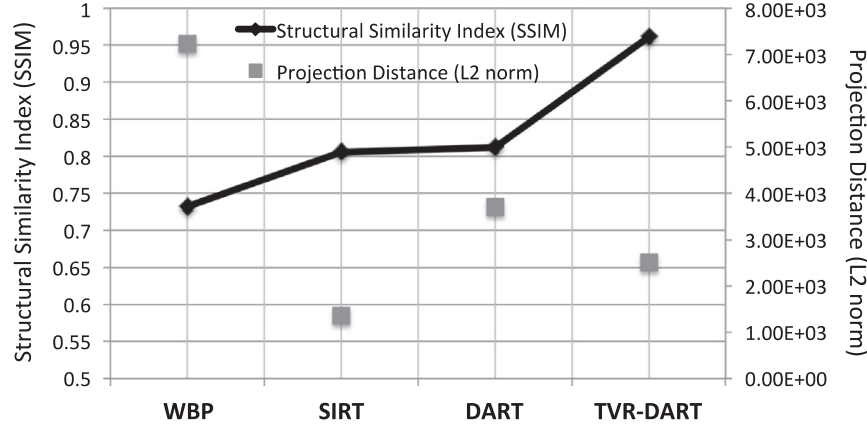

Fig. 9. Structural Similarity Index (SSIM) and Projection Distance $\left(\ell^{2}\right.$-norm) for reconstructions of the inorganic nanotube obtained using WBP, SIRT, DART and TVRDART from 42 projections ( $2.44^{\circ}$ tilt step). Corresponding reconstructions using 698 projections $\left(0.14^{\circ}\right.$ tilt step) were used as the reference images for the calculation of the SSIM. TVR-DART shows the highest structural similarity (0.961), while exhibiting the 2nd best match with the projection data.
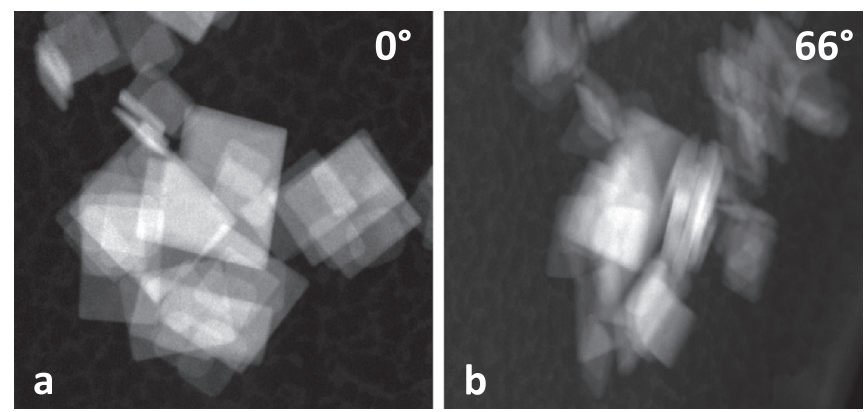

Fig. 10. HAADF-STEM images of anatase nanosheets recorded at tilt angles of (a) $0^{\circ}$ and (b) $+66^{\circ}$, taken from the complete $130^{\circ}$ tilt range.

contrast, DART and TVR-DART deliver improved reconstructions with reduced artifacts. DART still produces a hint of a false double layer structure, which is completely resolved in the TVR-DART results. Due to high noise level, a false rough surface on the inner and outer layer of the nanotube is also observed in the DART reconstruction. We further compare the reconstructions obtained using a drastically reduced dataset with only 42 projection images and a uniform $2.44^{\circ}$ tilt increment. In this case, WBP delivers a reconstruction with a much higher noise level while SIRT handles noise better but results in blurrier images. DART produces a noisier reconstruction, especially on the surface of the nanotube, while TVR-DART delivers the most consistent reconstruction when compared with the results obtained from the complete tilt series. This is confirmed in Fig. 9 where TVR-
DART shows a SSIM of 0.961 between reconstructions obtained with 698 and 42 projections. The value of the projection distance shows the 2nd lowest value among the four techniques. Under limited data, projection distance is no longer a reliable measure for the quality of reconstruction, but a quantitative indicator for the match between the reconstruction and the projection data. Therefore, under limited tilt range and/or number of projections, a blurred SIRT reconstruction can have better match with the dataset, therefore lower projection distance, but in fact far less accurate compared with advanced prior-based algorithms, such as TVR-DART.

\subsection{Anatase nanosheets acquired in dark-field (HAADF-STEM) mode}

Titanium dioxide $\left(\mathrm{TiO}_{2}\right)$ anatase nanosheets were prepared under hydrothermal conditions from the reagents titaniumbutoxide and hydrofluoric acid. Electron tomographic tilt series were acquired in HAADF-STEM mode by using a FEI Tecnai G2 TEM operated at $200 \mathrm{kV}$. A Fischione tilt-rotation tomography holder (model 2040) was used, with images acquired automatically using Xplore 3D software over a tilt range of $-70^{\circ}$ to $+66^{\circ}$ and a tilt increment of $2^{\circ}$. The pixel size of the projection images was $0.50 \mathrm{~nm}$. Alignment of the images was performed using Inspect 3D software. The first 3 projection images were removed from the recorded tilt series due to alignment issues, resulting in a final tilt range of $-64^{\circ}$ to $+66^{\circ}$ ( 66 projections in total). An intensity offset-correction was applied before performing tomographic reconstructions. Two of the projection images, which were acquired at tilt angles of $0^{\circ}$ and $+66^{\circ}$, are shown in Fig. 10. Each image shows a cluster of thin $\mathrm{TiO}_{2}$ nanosheets.

The signal recorded in HAADF-STEM mode is predominantly incoherent, with the contrast depending mainly on thickness and atomic number. However, as the nanosheets are agglomerated together, the total thickness of the specimen means that the projection requirement is only approximately fulfilled, which presents a challenge for conventional discrete tomography techniques. The flat horizontal structure of the nanosheets also represents one of the most difficult morphologies to recover under missing wedge conditions, as the majority of the Fourier representation of a horizontal structure is not sampled using a limited tilt range.

Fig. 11 shows the reconstructed cross-sections through the nanosheets obtained from both the full dataset and a reduced dataset in which the angular range was further reduced to $-60^{\circ}$ to $+60^{\circ}$ with a tilt increment of $4^{\circ}$ (31 projection images). Missing wedge artifacts are visible in the WBP and SIRT reconstructions where horizontal slabs are hardly identifiable that could severely hamper the quantification analysis. Here we have added a positive-constraint in the SIRT implementation that enforces positive intensities in the reconstruction.
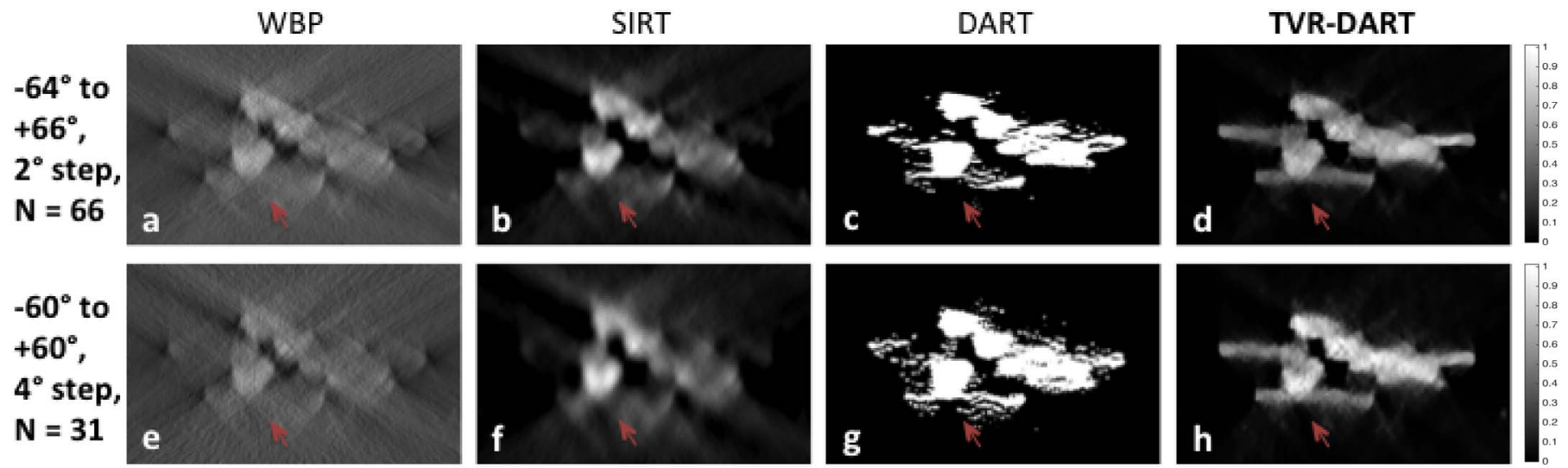

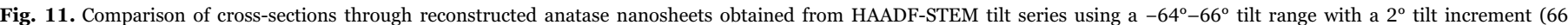

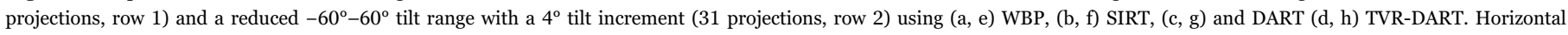

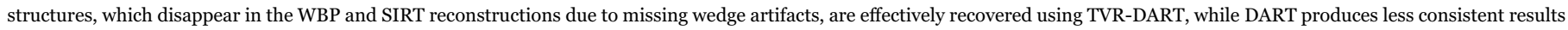
when the data condition changes. 


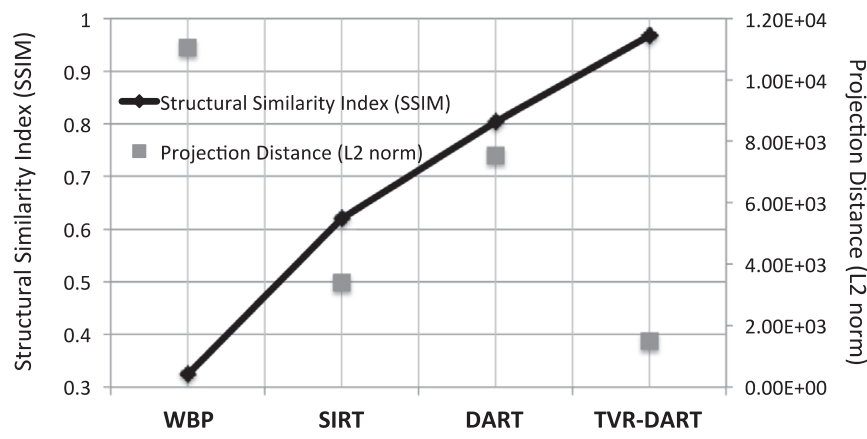

Fig. 12. Structural Similarity Index (SSIM) and projection distance $\left(\ell^{2}\right.$-norm) for reconstructions of the anatase nanosheets using WBP, SIRT, DART and TVR-DART using a $-60-60^{\circ}$ tilt range and a $4^{\circ}$ tilt increment (31 projections). Corresponding reconstructions from a $-64-66^{\circ}$ tilt range and a $2^{\circ}$ tilt increment (66 projections) were used as reference images for calculation of the SSIM. TVR-DART shows the highest structural similarity $(0.961)$, while exhibiting the best match with the projection data.

Despite slight improvements in contrast, horizontal structures are still greatly affected by limited tilt range. DART improves this by further recovering part of the horizontal structures but also produces some inconsistent artifacts around and between the nanosheets. TVR-DART delivers almost identical reconstructions under both full and reduced tilt series with well-recovered facets visible on the nanosheets. The computed SSIM (Fig. 12) is 0.966, demonstrating that accurate reconstructions can still be obtained using TVR-DART from a $\pm 60^{\circ}$ tilt range with a $4^{\circ}$ tilt increment. Furthermore, the reconstruction from TVR-DART is the most consistent with the acquired tilt series indicated by the lowest projection distance among all techniques.

\section{Conclusions}

In this paper, we have presented the application of a new iterative reconstruction technique, TVR-DART, for discrete electron tomography of nanomaterials. It is demonstrated via three experimental datasets that the proposed technique is capable of consistently delivering high-fidelity reconstructions with significantly reduced missing wedge artifacts under limited tilt range and a small number of projection images. With the needle-shaped polymer composite, we showed the successful application of discrete tomography for tilt series collected using bright-field TEM, and were able to experimentally verify the accuracy of the reconstruction under a $\pm 60^{\circ}$ tilt range by comparing it with the results from the $\pm 90^{\circ}$ full rotation. The tilt series of an inorganic nanotube recorded in $3.5 \mathrm{~s}$ using a direct electron detector helped demonstrate the ability of the proposed technique to cope with extremely high noise level in the projection data and verification of the reconstructions under more than an order of magnitude dose reduction. With the HAADF-STEM datasets from anatase nanosheets, the recoveries of the most challenging horizontal structures under limited tilt range are further verified. Taken together, these results provide strong evidence that the proposed discrete tomography technique can provide the electron microscopy community with a robust and automated tool for routine high-fidelity characterization of nanomaterials. The implementation of the proposed technique (in Python) is available in the GitHub repository of the ASTRA tomography toolbox via the following link:

https://github.com/astra-toolbox/ContributedTools/.

\section{Acknowledgments}

This work has been supported in part by the Stichting voor de Technische Wetenschappen (STW) through a personal grant (Veni,13610), and was in part by ExxonMobil Chemical Europe Inc. The authors further acknowledge financial support from the University of Antwerp through BOF GOA funding. S.B. acknowledges financial support from the European Research Council (ERC Starting Grant \#335078-COLOURATOMS). R.D.B. is grateful for funding from the European Research Council under the European Union's Seventh Framework Programme (FP7/2007-2013)/ ERC grant agreement number 320832. Thomas Altantzis is gratefully acknowledged for acquiring the Anatase nanosheets dataset.

\section{Appendix}

Here we demonstrate the common workflow for creating a 3D discrete tomographic reconstruction with the available TVR-DART python implementation by providing a code sample applied on the nanotube dataset in Section 3.2.

1. The projection data is first read into the python environment and stored as a numpy array. The tilt angles are also specified.

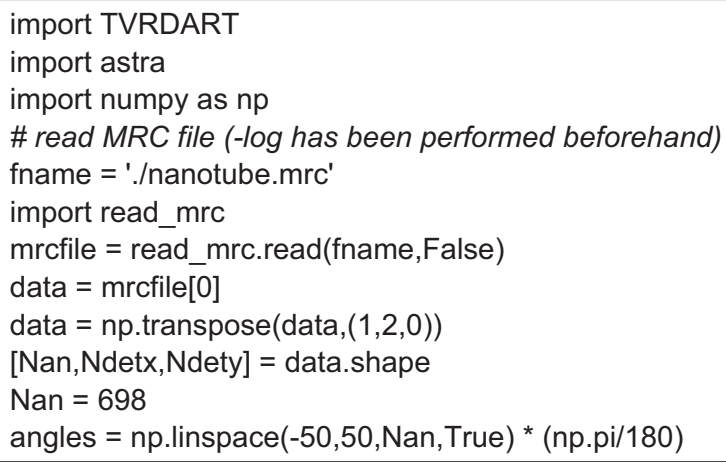

2. An intensity-offset correction is performed to make sure the average value of the visible background equals zero (corresponding to vacuum). Note that for bright-field data, negative logarithm is a necessary step before the intensity-offset correction. For this specific dataset, this has been done on the mrc file. 
background = data[int(np.round(Nan/2)), 274:330,80:150]

offset $=$ np. mean $($ background $)$

data $-=$ offset

3. The projection and volume geometry as well as operators are specified and created. Here we use the python interface of the ASTRA tomography toolbox to perform efficient forward and back projection operations using either CPU or GPU.

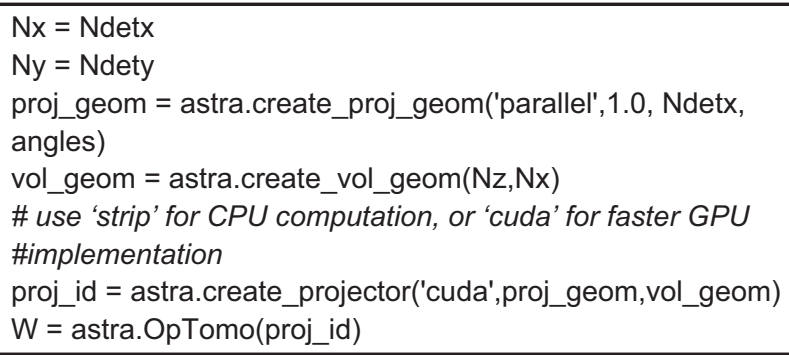

4. Initial reconstruction is performed with SIRT. The resulting 3D reconstruction and projection data is normalized by the maximum value of the initial reconstruction so that the value range lies approximately between 0 and 1 . This makes it easier to have a stable choice of $\lambda$ for various experimental datasets.

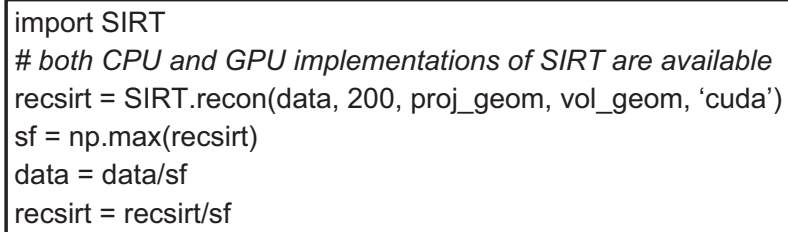

5. The parameters of TVR-DART are specified. This includes the number of material compositions in the specimen (including vacuum), $N_{g v}$, the sharpness of the soft segmentation function, $K$, the weight for the total variation term, $\lambda$, and the number of iterations, $N_{\text {iter }}$. A universal initial value for $K$ is 4 , and the algorithm will automatically adjust it.

$\mathrm{Ngv}=2$ \# number of material compositions in the specimen

$\mathrm{K}=4^{*} \mathrm{np}$.ones(Ngv-1) \# sharpness of soft segmentation function

lambda $=10 \#$ weight for TV term of the objective function

Niter $=50$ \# number of iterations

6. TVR-DART then automatically estimates the grey levels of the discrete reconstruction and adjusts some of the parameter settings. We do this using one slice or a few slices of the projection data (one slice means a detector row along the plane orthogonal to the tilt axis). Because we have normalized both our projection data and initial reconstruction, the initial values for grey levels can be set uniformly distributed between 0 and 1 . In difficult conditions where a really low contrast material is present, it can be helpful to use the average pixel values within identified regions of different materials from the normalized initial reconstruction.

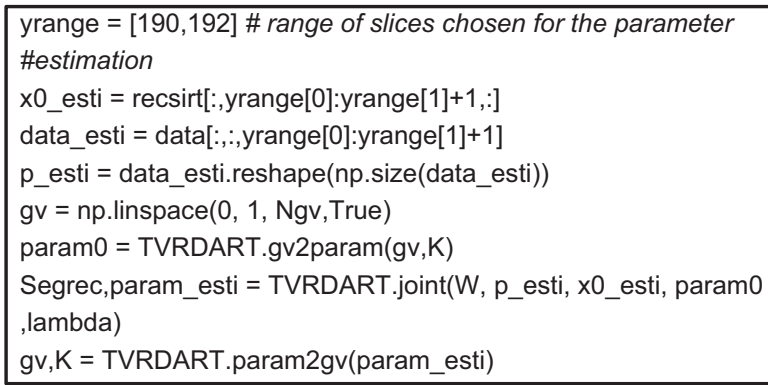


7. Finally, the TVR-DART reconstruction of the full 3D dataset is performed using the estimated parameters.

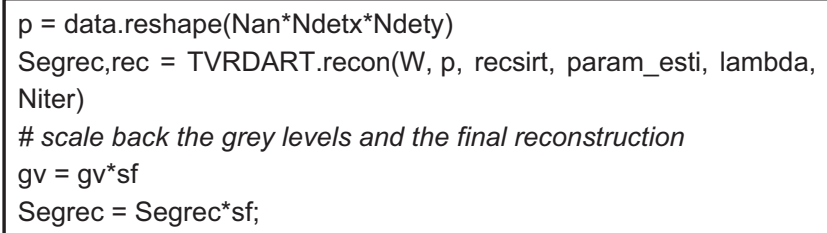

This example script is available at the GitHub repository of the ASTRA tomography toolbox.

\section{References}

[1] P.A. Midgley, R.E. Dunin-Borkowski, Electron tomography and holography in materials science, Nat. Mater. 8 (2009) $271-280$.

[2] S. Bals, G. Van Tendeloo, C. Kisielowski, A new approach for electron tomography: annular dark-field transmission electron microscopy, Adv. Mater. 18 (7) (2006) 892-895.

[3] H. Jinnai, X. Jiang, Electron tomography in soft materials, Curr. Opin. Solid State Mater. Sci. 17 (2013) $135-142$.

[4] H. Jinnai, R.J. Spontak, T. Nishi, Transmission electron microtomography and polymer nanostructures, Macromolecules 43 (2010) $1675-1688$.

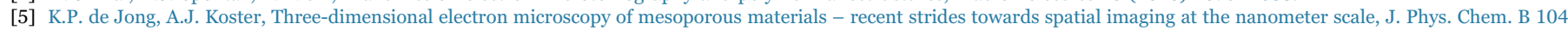
(2000) 9368.

[6] P.A. Midgley, M. Weyland, 3D electron microscopy in the physical sciences: the development of Z-contrast and EFTEM tomography, Ultramicroscopy 96 (2003) $413-431$.

[7] D.H. Anjum, R. Stiger, J. Finly, J.F. Conway, cryo-TEM of Ag nanoparticles grown on an ionic liquid, J. Mater. Res. 25 (7) (2010).

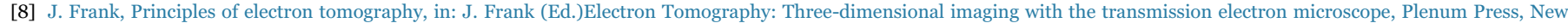
York, London, 1992, pp. 1-13.

[9] P. Penczek, M. Marko, K. Buttle, J. Frank, Ultramicroscopy 60 (1995) 393.

[10] H. Sugimori, T. Nishi, H. Jinnai, Dual-axis electron tomography for three-dimensional observations of polymeric nanostructures, Macromolecules 38 (2005) 10226-10233.

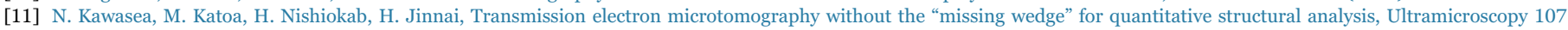
(2007) 8-15.

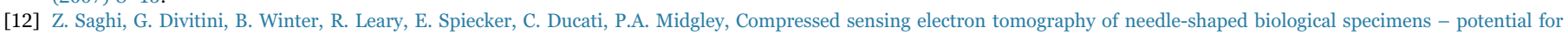
improved reconstruction fidelity with reduced dose, Ultramicroscopy 160 (2016) 230-238.

[13] X. Ke, S. Bals, D. Cott, T. Hantschel, H. Bender, G. Van Tendeloo, Three-dimensional analysis of carbon nanotube networks in interconnects by electron tomography without missing wedge artifacts, Microsc. Microanal. 16 (2010) 210-217.

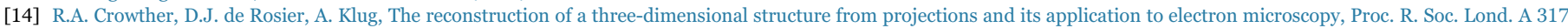
(1530) (1970) 319-340.

[15] P. Gilbert, Iteration methods for the three-dimensional reconstruction of an object from projections, J. Theor. Biol. 36 (1972) $105-107$.

[16] D.L. Donoho, Compressed sensing, IEEE Trans. Inf. Theory 52 (4) (2006) 1289-1306.

[17] R. Leary, Z. Saghi, P. Midgley, D.J. Holland, Compressed sensing electron tomography, Ultramicroscopy 131 (2013) $70-91$.

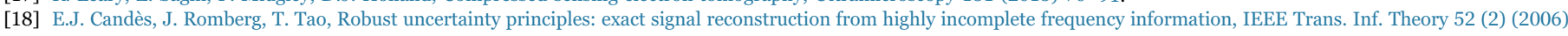
489-509.

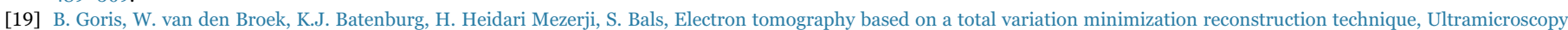
113 (2012) 120-130 (Feb.).

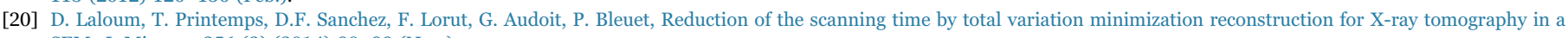
SEM, J. Microsc. 256 (2) (2014) 90-99 (Nov.).

[21] G.T. Herman, A. Kuba, Advances in discrete tomography and its applications, Birkhäuser Basel, 2007.

[22] S. Van Aert, K.J. Batenburg, M.D. Rossell, R. Erni, G. Van Tendeloo, Three-dimensional atomic imaging of crystalline nanoparticles, Nature 470 (2011) 374-377.

[23] K.J. Batenburg, J. Sijbers, DART: a practical reconstruction algorithm for discrete tomography, IEEE Trans. Image Process. 20 (2011) $2542-2553$ (Sept.).

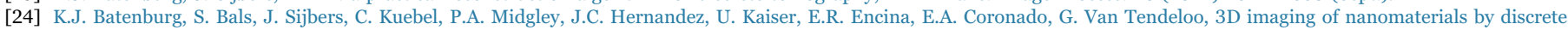
tomography, Ultramicroscopy 109 (6) (2009) 730-740.

[25] S. Bals, K.J. Batenburg, J. Verbeeck, J. Sijbers, G. Van Tendeloo, Quantitative three-dimensional reconstruction of catalyst particles for bamboo-like carbon nanotubes, Nano Letters 7 (12) (2007) 3669-3674.

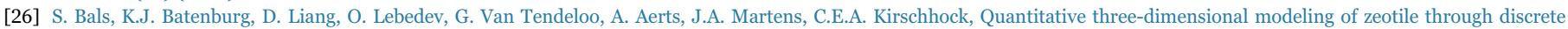
electron tomography, J. Am. Chem. Soc. 131 (13) (2009) 4769-4773.

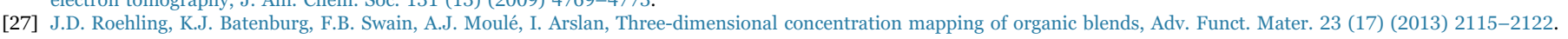

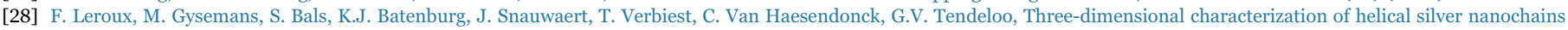
mediated by protein assemblies, Adv. Mater. 22 (19) (2010) 2193-2197.

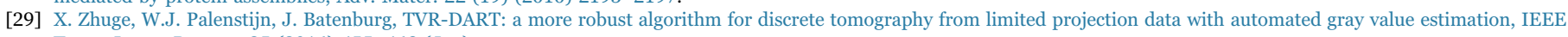
Trans. Image Process. 25 (2016) 455-468 (Jan).

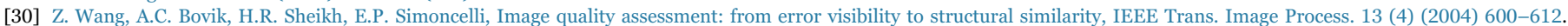

[31] L.S. Panchakarla, et al., Lanthanide-based functional misfit-layered nanotubes, Angew. Chem. Int. Ed. 53 (2014) 6920-6924. http://dx.doi.org/10.1002/anie.201404189.

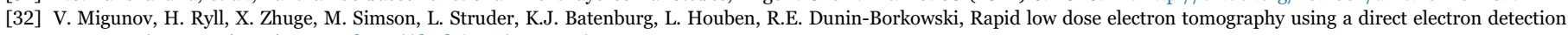
camera, Sci. Rep. 5 (2015) 14516. http://dx.doi.org/10.1038/srep14516. 